\title{
The Composition-Tunable Polydiacetylenic Complex Films: Conformational Change upon Thermal Stimulation and Preferential Interaction with Specific Small Molecules
}

\author{
Chao Wang, ${ }^{1}$ Jin Hyuk Park, ${ }^{2}$ Songwen Tan, ${ }^{3}$ Chunzhi Cui, ${ }^{1,2}$ \\ Jing-Yi Jin, ${ }^{1}$ and Dong June $\mathrm{Ahn}^{2,3,4}$ \\ ${ }^{1}$ Department of Chemistry, College of Science and Key Laboratory of Natural Resources of \\ Changbai Mountains \& Functional Molecules, Ministry of Education, Yanbian University, Yanji 133002, China \\ ${ }^{2}$ Department of Chemical and Biological Engineering, Korea University, Seoul 02841, Republic of Korea \\ ${ }^{3}$ Department of Bio-Microsystem Technology, Korea University, Seoul 02841, Republic of Korea \\ ${ }^{4}$ KU-KIST Graduate School of Converging Science and Technology, Korea University, Seoul 02841, Republic of Korea
}

Correspondence should be addressed to Chunzhi Cui; cuichunzhi@ybu.edu.cn, Jing-Yi Jin; jyjin-chem@ybu.edu.cn, and Dong June Ahn; ahn@korea.ac.kr

Received 1 February 2017; Revised 17 April 2017; Accepted 27 April 2017; Published 24 May 2017

Academic Editor: Surinder Singh

Copyright (c) 2017 Chao Wang et al. This is an open access article distributed under the Creative Commons Attribution License, which permits unrestricted use, distribution, and reproduction in any medium, provided the original work is properly cited.

Polydiacetylenic complex films were prepared using 10,12-pentacosadiynoic acid (PCDA) and para-xylenediamine ( $p$ XDA) upon acid-base interactions. The thermochromatic reversibility of the complex films was modulated by changing the mixed molar ratio $(3: 1,2: 1$, and $1: 1)$ of the two molecules. The corresponding conformational changes of the complex films were studied by ex situ FTIR analysis upon thermal stimulation for the first time. In addition, the binding specificities of $\alpha$-, $\beta$-, and $\gamma$-cyclodextrins (CDs) with the films were studied, where the $\alpha$-CDs can induce stronger red fluorescent emission of the films. These fundamental results may be useful for platforms that use these polydiacetylenic complex films as optoelectronic devices or chemical/biological sensors.

\section{Introduction}

Conjugated polymers are well-known sensing materials because of the changes in their electronic absorption and photon emission responding to environmental perturbations $[1,2]$. Based on this property, a variety of conjugated polymers, such as polythiophene [3-5], polyaniline $[6,7]$, polypyrrole [8], polyacetylene [9], and polydiacetylene (PDA) [10-12], have been investigated as sensing materials. Though all of these conjugated polymers can be used as sensing materials, PDA is of significant interest due to its facile fabrication method for photopolymerization of wellordered diacetylene (DA) monomers [13].

Another unique property of PDA materials is their color change from blue to red, where the red phase of the PDA is fluorescent while the blue phase is not $[14,15]$, in response to various stimuli, including temperature [16, 17], organic solvent $[18,19]$, mechanical stress $[20,21]$, and ligandreceptor interactions $[22,23]$. The phase change from blue to red is due to the release of side-chain strain. The partial distortion of conjugated p-orbital arrays shortens the effective $\pi$-conjugated length, which changes the electronic states and the optical properties [24-26]. The blue-to-red color change of PDA is generally not reversed after removing the external stimuli, though a few examples of reversible chromism in specially designed PDA materials have been reported to date [16, 27]. Recently, we synthesized PDA complex films using two organic molecules at different ratios, 10,12-pentacosadiynoic acid (PCDA) and para-xylenediamine ( $p \mathrm{XDA})$ [28]. These complex films were formed by acid-base interactions between the carboxylic acid groups of PCDA and the amino groups of $p \mathrm{XDA}$ as well as proton transfer from the carboxylic 
acid to the amino group. These polydiacetylenic complex films are phase-reversible upon thermal stimulation. Their modulated reversibility is related to the intermolecular $\pi-\pi$ stacking. However, a detailed analysis has not been carried out yet to study the phase-reversing mechanism and related conformational changes of the complex films.

Here, the influence of conformational changes on the thermochromism of the complex films has been studied during phase transition and reversing using ex situ FTIR spectroscopic analysis to reveal the detailed interactions among the bimolecules. In addition, as part of our continuing efforts to develop polydiacetylenic complex films, we studied cyclodextrins- (CDs-) induced color transitions and the fluorescent properties of the films.

\section{Experimental}

2.1. Fabrication of the Polydiacetylenic Complex Films. A detailed fabrication method for polydiacetylenic complex films has been described in previous reports [28, 29]. Firstly, PCDA and $p$ XDA were dissolved in tetrahydrofuran (THF) at a concentration of $1.5 \times 10^{-2} \mathrm{M}$, respectively. Then different molar ratios $(3: 1,2: 1$, and $1: 1)$ of the mixture solution were used to form thin films by spin-coating on a calcium fluoride $\left(\mathrm{CaF}_{2}\right)$ substrate. The resulting white films on $\mathrm{CaF}_{2}$ substrates were photopolymerized at $254 \mathrm{~nm}$ for $2 \mathrm{~min}$ to form polymeric PDA complex films in blue phase. Red phase PDA complex films were obtained from the blue phase PDA complex films by applying thermal stimuli.

2.2. Characterization. The complex films were visualized with a scanning electron microscope (SEM; JEOL, JSM-5200) and a high-resolution transmission electron microscope (HR-TEM; JEOL, JEM-3010). The structure of the films was characterized by X-ray scattering (Bruker D8 DISCOVER). An FTIR spectrometer (Perkin-Elmer, Spectrum GX) was used to acquire transmission spectra for the films spincoated on the $\mathrm{CaF}_{2}$ substrate. A mercury cadmium tellurium (MCT) detector was cooled with liquid nitrogen. The detector collected 256 scans at a resolution of $4 \mathrm{~cm}^{-1}$. A nonpolarized infrared beam was used. The ex situ transmission spectra were obtained after the samples underwent a heating-andcooling cycle. The temperature was controlled to reach $65^{\circ} \mathrm{C}$, room temperature (RT), $85^{\circ} \mathrm{C}, \mathrm{RT}, 105^{\circ} \mathrm{C}, \mathrm{RT}, 125^{\circ} \mathrm{C}, \mathrm{RT}$, $145^{\circ} \mathrm{C}, \mathrm{RT}, 165^{\circ} \mathrm{C}$, and RT, in turn. The desired temperature was maintained for $10 \mathrm{~min}$ via a house-built temperaturecontrolled heating plate. The visible absorption spectra of the films were obtained using a UV-vis spectrophotometer (Agilent Technologies, HP 8453). The fluorescent images of the films were recorded using a fluorescence microscope (Olympus, BX51).

\section{Results and Discussion}

3.1. SEM, TEM, and XRD of Films. For morphological analysis, we employed SEM and HR-TEM. As shown in the SEM (Figures 1(a)-1(c)) and the magnified HR-TEM (Figures 1(d)-1(f)) images, all the complex films were composed of relative uniform patches ranging over several hundred nanometers. X-ray diffraction analysis confirmed the formation of highly ordered lamellar crystal structures with an intense (100) reflection as shown in Figure $1(\mathrm{~g})$. The crystal plane distance of the complex films was of $4.7 \mathrm{~nm}$. The other crystal planes are shown in the magnified X-ray diffraction patterns (see SI in Supplementary Material available online at https://doi.org/10.1155/2017/5983106).

3.2. FTIR Spectroscopic Analysis. In our previous work, we studied the visible color changes of polydiacetylenic complex films upon consecutive heating-and-cooling cycles for thermal reversibility analysis [28]. Ex situ FTIR analysis of the films was carried out in the transmission mode here to gain insight into the thermally promoted colorimetric reversibility of the three kinds of complex films. Figure 2 shows the ex situ FTIR spectra of the case of 2:1 (stoichiometric) film. The peaks observed at $1692,1644,1528,1460$, and $1408 \mathrm{~cm}^{-1}$ correspond to the $\mathrm{C}=\mathrm{O}$ stretching, $\mathrm{NH}_{3}{ }^{+}$asymmetric deformation, $\mathrm{COO}^{-}$asymmetric, $\mathrm{CH}_{2}$ scissoring, and $\mathrm{COO}^{-}$symmetric stretching bands, respectively. Initially, at RT, there was no $\mathrm{C}=\mathrm{O}$ stretching band but rather $\mathrm{COO}^{-}$asymmetric and symmetric stretching bands. This means that there is complete acid-base complexation between the carboxylic acid group of PCDA molecules and the amino group of pXDA molecules [29]. This complexation between the two molecules plays an important role in the conformational change upon thermochromism. By heating and cooling the $\mathrm{CaF}_{2}$ substrate, we observed the FTIR spectra at RT. No significant changes were observed in the FTIR spectra after the complex films underwent $65^{\circ} \mathrm{C}$-RT, $85^{\circ} \mathrm{C}$-RT, and $105^{\circ} \mathrm{C}$ RT cycles. During the heating process, the films turned deep blue $\left(65^{\circ} \mathrm{C}\right)$, purple $\left(85^{\circ} \mathrm{C}\right)$, and $\operatorname{red}\left(105^{\circ} \mathrm{C}\right)$. They all return to the initial blue color after cooling to RT (see SI). The color change due to heating is likely polymer side-chain reordering. This decreases the polymer effective conjugation length. However, in this system, $\pi-\pi$ stacking between the $p$ XDA molecules makes the conformational structure of PDA return to the initial form. However, after undergoing $125^{\circ} \mathrm{C}$-RT cycle, the peak of the $\mathrm{C}=\mathrm{O}$ stretching band from the carboxyl acid group was observed at $1692 \mathrm{~cm}^{-1}$ and the intensities of $\mathrm{NH}_{3}{ }^{+}$asymmetric deformation and $\mathrm{COO}^{-}$asymmetric and symmetric stretching bands decreased. This means that some complexation in the complex films was destroyed, and some PDA molecules were isolated from the pXDA molecules. This was followed by $145^{\circ} \mathrm{C}$-RT and $165^{\circ} \mathrm{C}$-RT cycles, and the intensity of the $\mathrm{C}=\mathrm{O}$ stretching band gradually increased. The intensities of the $\mathrm{NH}_{3}{ }^{+}$asymmetric deformation and $\mathrm{COO}^{-}$ asymmetric and symmetric stretching bands continuously decreased. As shown in the photographs, the colors of the films turned purple and red sequentially after cooling (see SI). Meanwhile, we can observe that the peak of $\mathrm{C}=\mathrm{O}$ stretching band broadens, and the shoulder peak at $1712 \mathrm{~cm}^{-1}$ corresponds to the $\mathrm{C}=\mathrm{O}$ stretching of red phase PDA molecules [30].

Figure 3 shows the ex situ FTIR spectra of the case of $3: 1$ (termed as PCDA-rich) film. Initially, the spectra show the $\mathrm{C}=\mathrm{O}$ stretching band as well as the $\mathrm{NH}_{3}{ }^{+}$asymmetric 


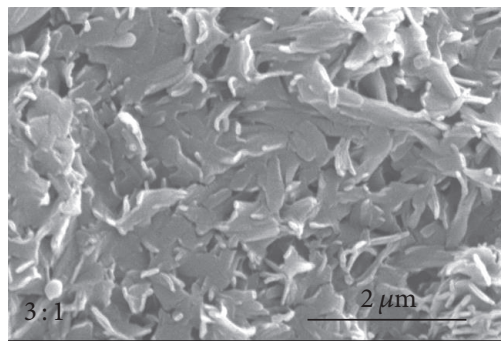

(a)

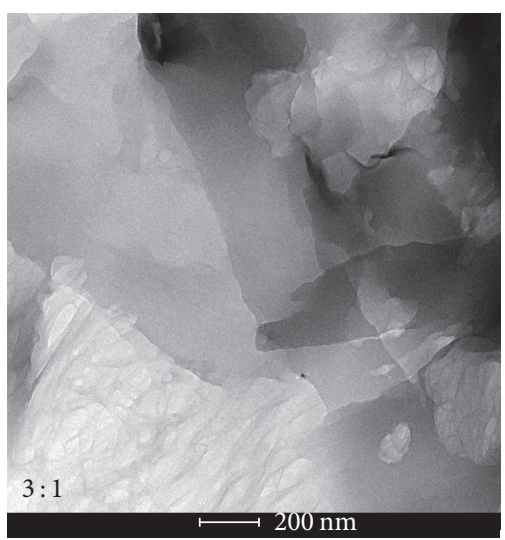

(d)

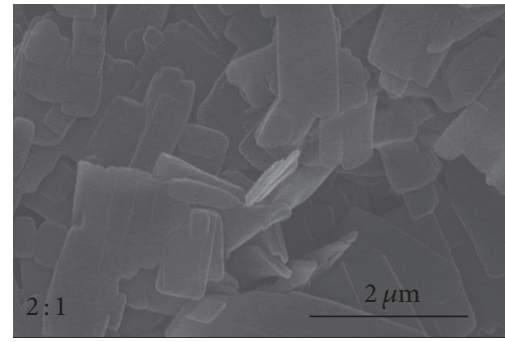

(b)

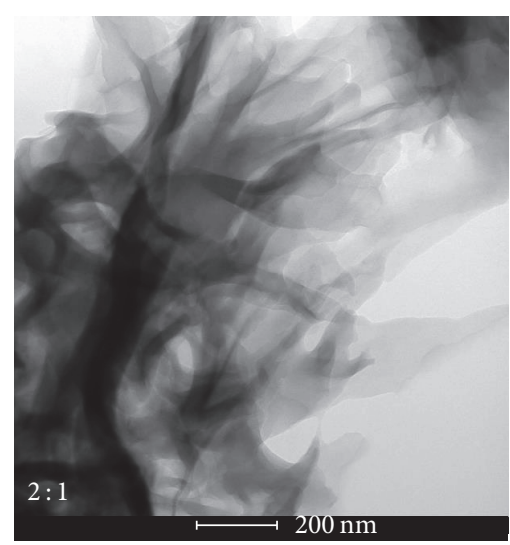

(e)

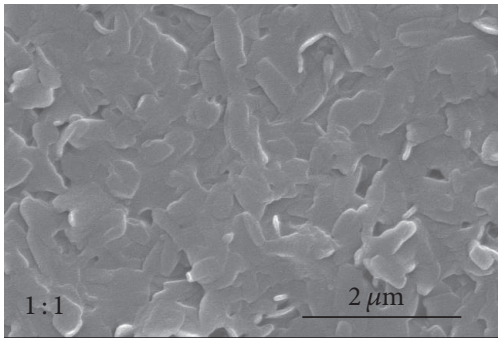

(c)

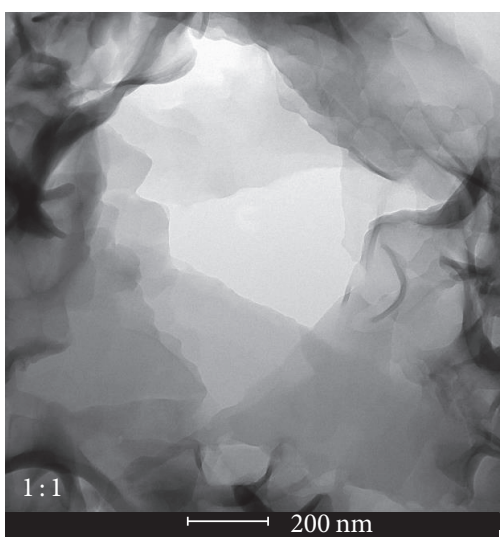

(f)

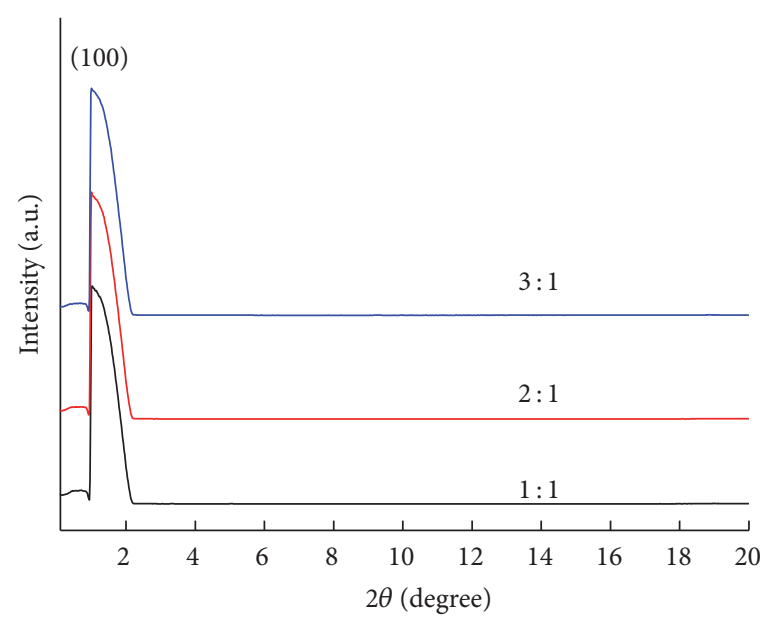

(g)

Figure 1: SEM (a)-(c) and HR-TEM (d)-(f) images and X-ray diffraction patterns (g) for 3:1, 2:1, and 1:1 polydiacetylenic films.

deformation, $\mathrm{COO}^{-}$asymmetric and symmetric stretching bands, and $\mathrm{CH}_{2}$ scissoring. These observations indicate the coexistence of complexed and noncomplexed PDA molecules. Hence, after undergoing the $65^{\circ} \mathrm{C}$-RT cycle, the intensity of $\mathrm{C}=\mathrm{O}$ stretching band decreased and shifts to $1712 \mathrm{~cm}^{-1}$ due to the noncomplexed PDA molecules. There are also some red phases in the film (see SI). Following the next $85^{\circ} \mathrm{C}$-RT, $125^{\circ} \mathrm{C}$-RT, and $125^{\circ} \mathrm{C}$-RT cycles, the peak at $1712 \mathrm{~cm}^{-1}$ gradually increased, and this was accompanied by increase of the red phase in the film. After $145^{\circ} \mathrm{C}$-RT and $165^{\circ} \mathrm{C}-\mathrm{RT}$ cycles, the intensity of the $\mathrm{C}=\mathrm{O}$ stretching band at $1692 \mathrm{~cm}^{-1}$ slightly increases accompanied by the intensity of the $\mathrm{NH}_{3}^{+}$asymmetric deformation and the $\mathrm{COO}^{-}$ asymmetric and symmetric stretching bands decrease. The phenomenon of $3: 1$ film is similar to the case of $2: 1$ film from the $145^{\circ} \mathrm{C}$-RT cycle.

Finally, the 1:1 complex film showed the strongest reversibility upon thermal stimulation. The ex situ FTIR spectra of 


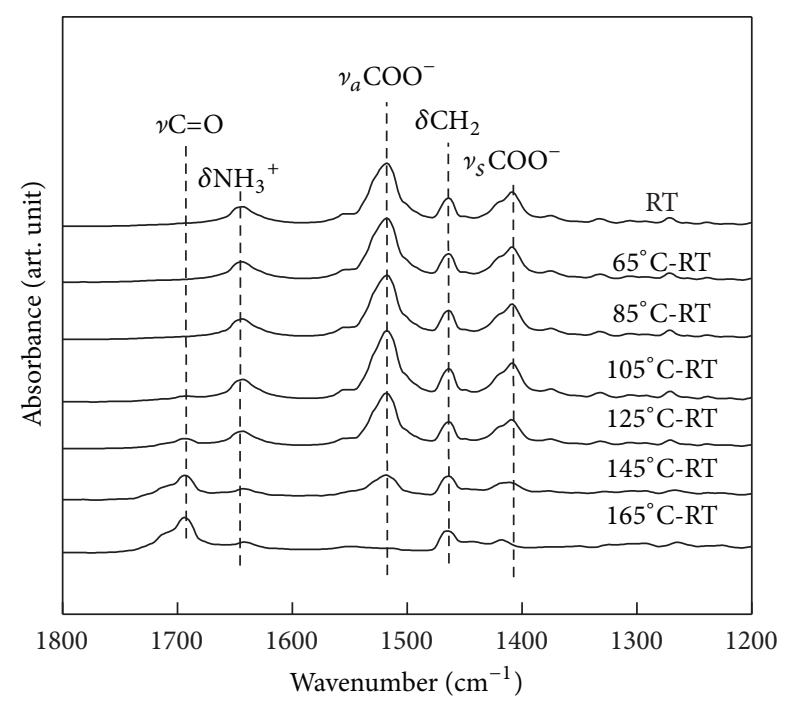

FIGURE 2: Ex situ FTIR spectra of a 2:1 polydiacetylenic film upon thermal stimulus.

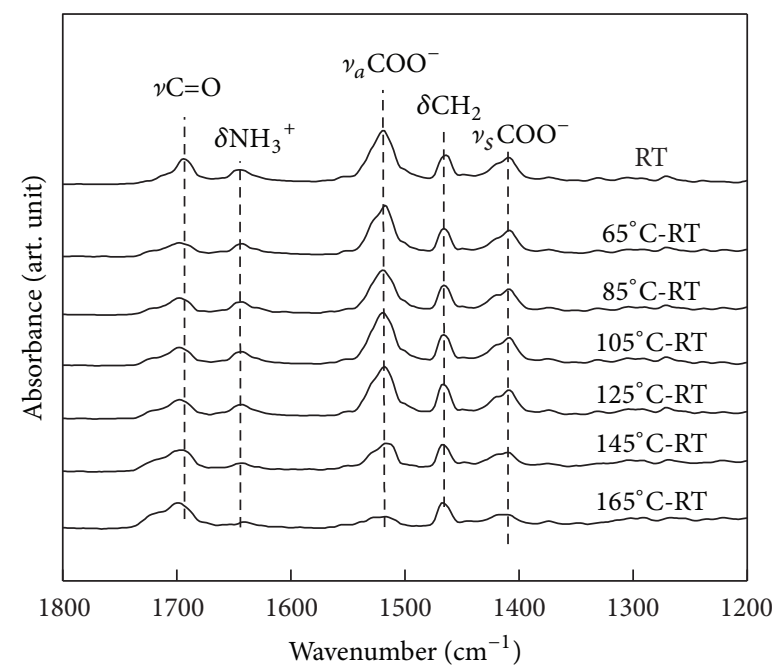

FIGURE 3: Ex situ FTIR spectra of a 3:1 polydiacetylenic film upon thermal stimulus.

the 1:1 (termed as $p$ XDA-rich) film upon thermal stimulation are shown in Figure 4. Due to enlarged reversibility window for $2: 1$ and $3: 1$ films, there are no evident changes in the spectra until the $125^{\circ} \mathrm{C}$-RT cycle; that is, the film remains blue (see SI). After the film undergoes the $145^{\circ} \mathrm{C}$-RT cycle, the intensities of the $\mathrm{NH}_{3}{ }^{+}$asymmetric deformation and $\mathrm{COO}^{-}$ asymmetric and symmetric stretching bands decrease. The $\mathrm{C}=\mathrm{O}$ stretching band at $1692 \mathrm{~cm}^{-1}$ appears, and the color of the film remains. Ultimately, after the $165^{\circ} \mathrm{C}$-RT cycle, the film turns red, and the intensity of the $\mathrm{NH}_{3}{ }^{+}$asymmetric deformation disappeared similar to the $\mathrm{COO}^{-}$asymmetric and symmetric stretching bands. We consider the increased $\pi-\pi$ stacking to be due to high amount of $p$ XDA molecules, which results in the strongest thermochromism reversibility.

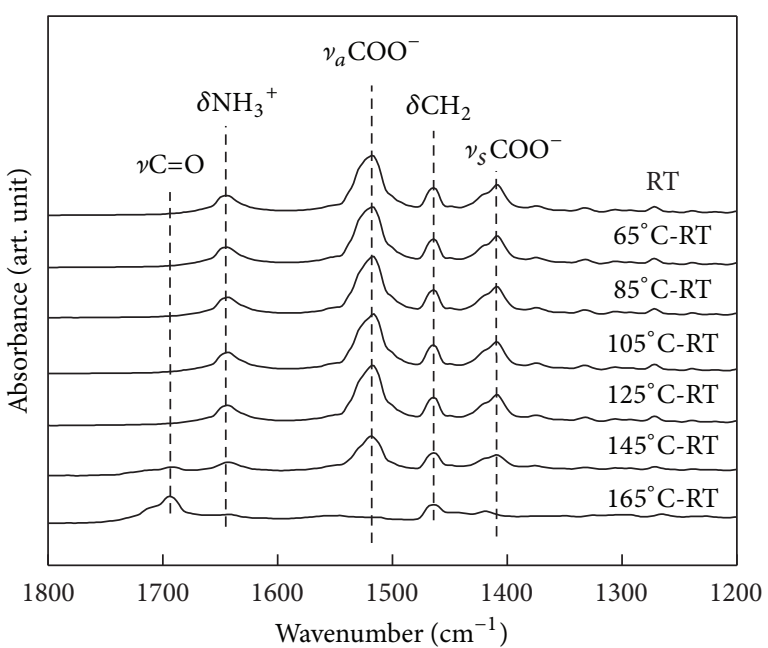

FIGURE 4: Ex situ FTIR spectra of a 1:1 polydiacetylenic film upon thermal stimulus.

We used FTIR to confirm the structural change of the films upon thermal stimulation as a function of molar ratio.

3.3. Interaction with CD Molecules. CDs are well known for their ability to form inclusion complexes with a variety of substrates [31]. In addition, different binding specificities of $\alpha$-, $\beta$-, and $\gamma$-CDs make these small molecules attractive model systems for studying ligand-receptor interactions. There have also been some reports about vesicles or Langmuir-Schaefer film type PDA materials that interact with CDs. Here, the optical changes of the $2: 1$ complex film upon interaction with different sizes of CDs were studied for the first time. The $2: 1$ complex film was incubated in an appropriate concentration of $\alpha-, \beta$-, and $\gamma$-CDs solution for $20 \mathrm{~min}$. The color changes of each film were monitored by UV-visible spectroscopy (Figure 5). Initially, the film shows a typical blue color corresponding to an absorption maximum wavelength of $640 \mathrm{~nm}$ on UV-vis spectra. After incubation in $1 \mathrm{mM}$ of $\alpha$-CDs solution, the absorption maximum peak remains at $640 \mathrm{~nm}$. However, the absorption maximum peak shifted to $540 \mathrm{~nm}$ after incubation with $5 \mathrm{mM}$ and $10 \mathrm{mM} \alpha$-CDs solution as shown in Figure 5(a). Compared with $\alpha$-CDs, neither $\beta$-CDs nor $\gamma$-CDs induced an absorption peak shift in the PDA films. As the concentration increases to $10 \mathrm{mM}$, both $\beta$-CDs and $\gamma$-CDs showed a small peak at $540 \mathrm{~nm}$ (Figures $5(\mathrm{~b})$ and 5(c)). A well-defined equation was used to quantify the colorimetric response (CR) [32]. Figure 5(d) shows that the CR values of the complex film caused by $\alpha-, \beta-$, and $\gamma$-CDs are $9.1 \%(1 \mathrm{mM}), 61.9 \%(5 \mathrm{mM})$, and $75.1 \%(10 \mathrm{mM})$, respectively. All of the CR values caused by $\beta$ - and $\gamma$-CDs are below $20 \%$. We also observed the fluorescent images of the same films (Figure 6). The films incubated in 5 and $10 \mathrm{mM} \alpha$ CDs solutions emit a strong red fluorescent signal. However, the films incubated in $\beta$ - and $\gamma$-CDs solutions only emit pale fluorescent signals. This suggests a shape match between the CDs and PDA-pXDA molecules. The $\alpha$-CDs respond to the 


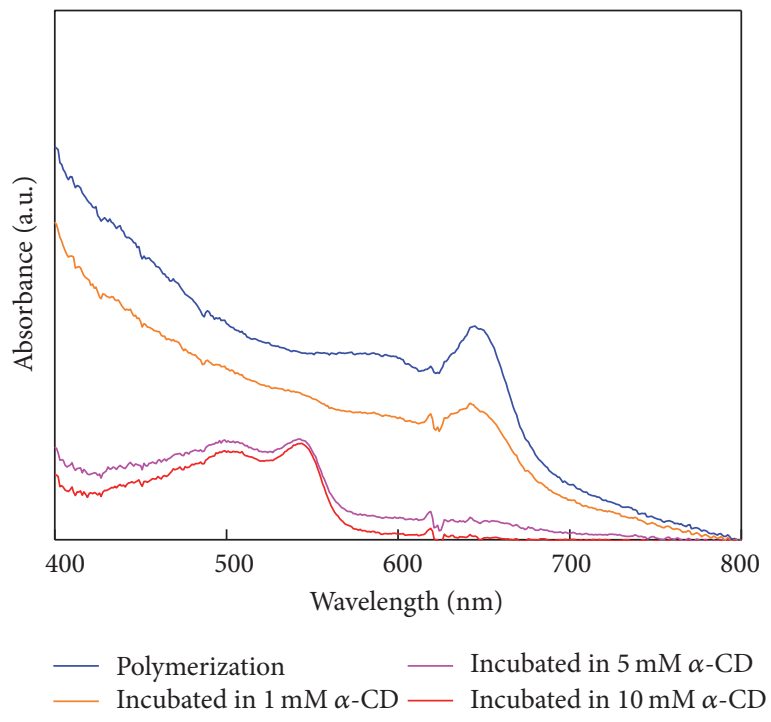

(a)

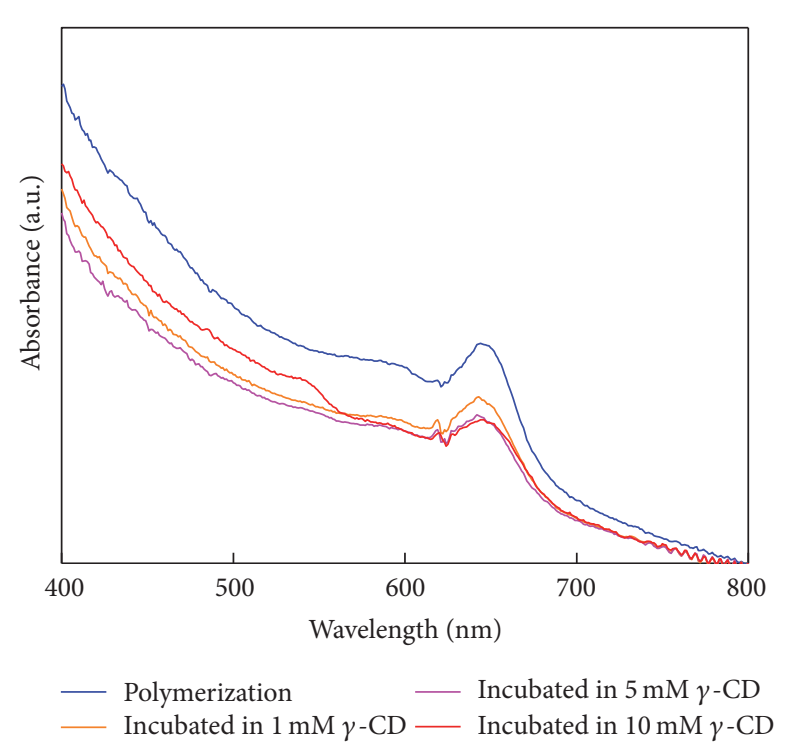

(c)

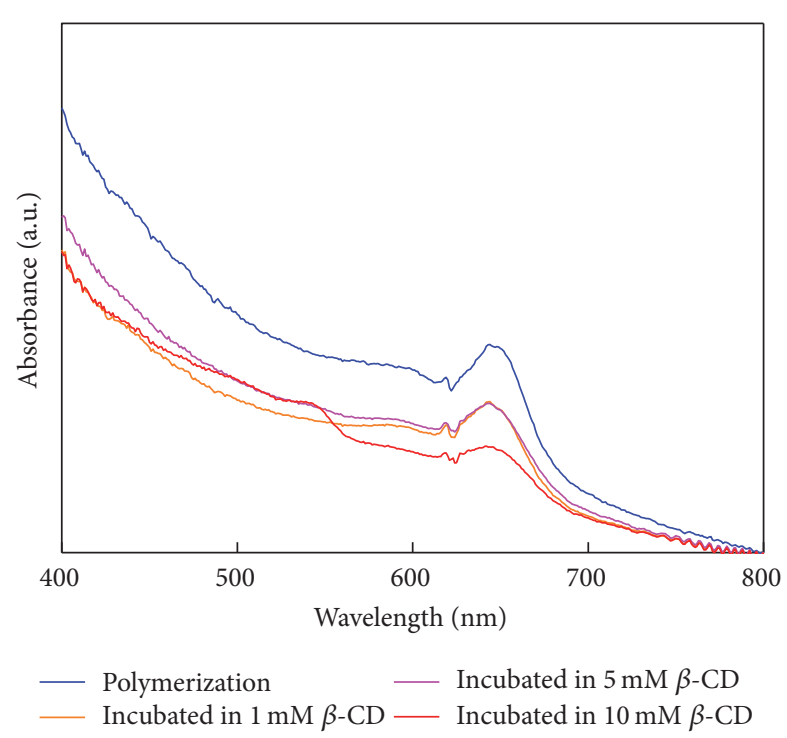

(b)

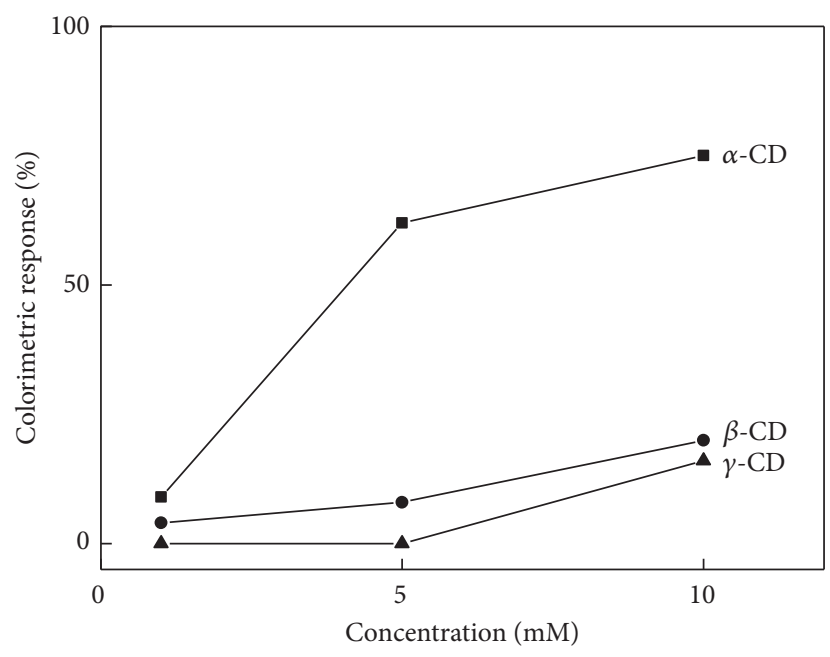

(d)

FIGURE 5: UV-visible absorption spectra of the films after 20 min incubation with various concentrations of $\alpha$ - (a), $\beta$ - (b), and $\gamma$-CDs (c) and the corresponding colorimetric response (CR) analysis (d).

best shape match and induce an irreversible phase transition of the complex films.

\section{Conclusions}

In summary, the composition-tunable polydiacetylenic complex films were successfully fabricated through acid-base interactions between PCDA and pXDA molecules. Conformational changes of the complex films upon thermal stimulation were observed by ex situ FTIR analysis. The thermochromism reversibility of the complex films was strongly dependent on the $\pi-\pi$ stacking between the pXDA molecules. We also observed the first example of a CDsinduced color transition and fluorescent emission of the complex films. These fundamental results help explain how $\pi-\pi$ stacking dependent on conformational changes affects the reversibility window and the development of a versatile sensor platform based on polydiacetylenic complex films.

\section{Conflicts of Interest}

The authors declare that there are no conflicts of interest regarding the publication of this paper. 

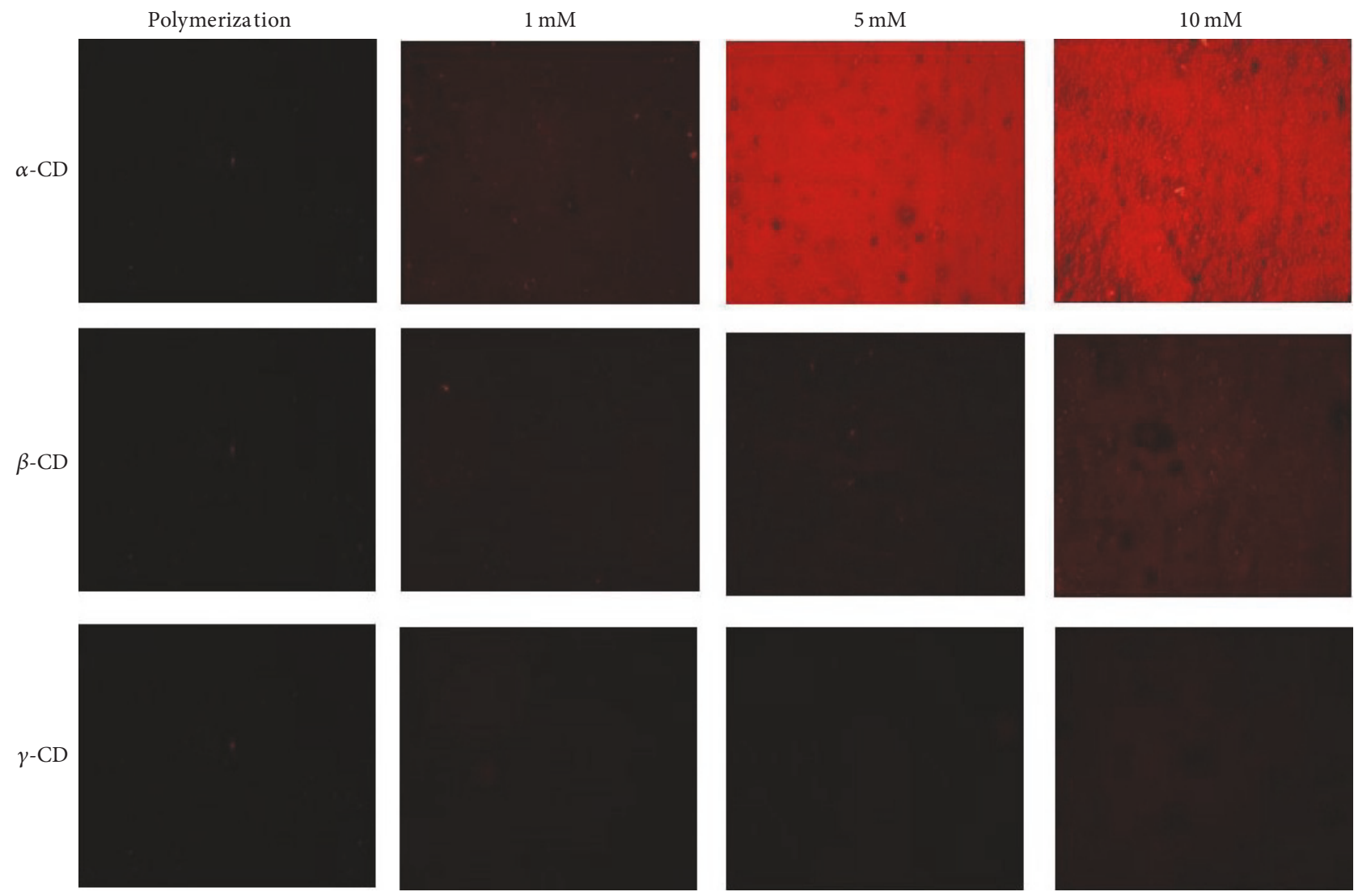

FIGURE 6: Fluorescent images of the films after 20 min incubation with various concentrations of $\alpha$-, $\beta$-, and $\gamma$-CDs. The exposure time was fixed at $0.5 \mathrm{~s}$.

\section{Authors' Contributions}

Chao Wang, Jin Hyuk Park, and Songwen Tan contributed equally to this work.

\section{Acknowledgments}

This work is supported by the National Research Foundation (NRF, 2015M3C1A3002152) and a Korea University grant.

\section{References}

[1] L. Feng, C. Zhu, H. Yuan, L. Liu, F. Lv, and S. Wang, "Conjugated polymer nanoparticles: preparation, properties, functionalization and biological applications," Chemical Society Reviews, vol. 42, no. 16, pp. 6620-6633, 2013.

[2] D. Tyler McQuade, A. E. Pullen, and T. M. Swager, "Conjugated polymer-based chemical sensors," Chemical Reviews, vol. 100, no. 7, pp. 2537-2574, 2000.

[3] C. Cui, D. H. Park, H. Choi, J. Joo, and D. J. Ahn, "Protein recognition by phase transition of aptamer-linked polythiophene single nanowire," Small, vol. 12, no. 9, pp. 1154-1158, 2016.

[4] T. Cassagneau and F. Caruso, "Inverse opals for optical affinity biosensing," Advanced Materials, vol. 14, no. 22, pp. 1629-1633, 2002.
[5] D. H. Park, M. S. Kim, and J. Joo, "Hybrid nanostructures using $\pi$-conjugated polymers and nanoscale metals: synthesis, characteristics, and optoelectronic applications," Chemical Society Reviews, vol. 39, no. 7, pp. 2439-2452, 2010.

[6] E. Shoji and M. S. Freund, "Potentiometric sensors based on the inductive effect on the $\mathrm{p} K_{a}$ of poly(aniline): a nonenzymatic glucose sensor," Journal of the American Chemical Society, vol. 123, no. 14, pp. 3383-3384, 2001.

[7] J. X. Huang, S. Virji, B. H. Weiller, and R. B. Kaner, "Polyaniline nanofibers: facile synthesis and chemical sensors," Journal of the American Chemical Society, vol. 125, no. 2, pp. 314-315, 2003.

[8] H. Korri-Youssoufi and A. Yassar, "Electrochemical probing of DNA based on oligonucleotide-functionalized polypyrrole," Biomacromolecules, vol. 2, no. 1, pp. 58-64, 2001.

[9] Y. Liu, R. C. Mills, J. M. Boncella, and K. S. Schanze, "Fluorescent polyacetylene thin film sensor for nitroaromatics," Langmuir, vol. 17, no. 24, pp. 7452-7455, 2001.

[10] S. Y. Okada, R. Jelinek, and D. Charych, "Induced color change of conjugated polymeric vesicles by interfacial catalysis of phospholipase $\mathrm{A}_{2}$," Angewandte Chemie-International Edition, vol. 38, no. 5, pp. 655-659, 1999.

[11] Q. Cheng and R. C. Stevens, "Coupling of an induced fit enzyme to polydiacetylene thin films: colorimetric detection of glucose," Advanced Materials, vol. 9, no. 6, pp. 481-483, 1997.

[12] D. H. Charych, J. O. Nagy, W. Spevak, and M. D. Bednarski, "Direct colorimetric detection of a receptor-ligand interaction 
by a polymerized bilayer assembly," Science, vol. 261, no. 5121, pp. 585-588, 1993.

[13] J. A. Dong and J.-M. Kim, "Fluorogenic polydiacetylene supramolecules: immobilization, micropatterning, and application to label-free chemosensors," Accounts of Chemical Research, vol. 41, no. 7, pp. 805-816, 2008.

[14] J. Wu, A. Zawistowski, M. Ehrmann, T. Yi, and C. Schmuck, "Peptide functionalized polydiacetylene liposomes act as a fluorescent turn-on sensor for bacterial lipopolysaccharide," Journal of the American Chemical Society, vol. 133, no. 25, pp. 9720-9723, 2011.

[15] J. Lee, H.-J. Kim, and J. Kim, "Polydiacetylene liposome arrays for selective potassium detection," Journal of the American Chemical Society, vol. 130, no. 15, pp. 5010-5011, 2008.

[16] D. J. Ahn, E.-H. Chae, G. S. Lee et al., "Colorimetric reversibility of polydiacetylene supramolecules having enhanced hydrogenbonding under thermal and $\mathrm{pH}$ stimuli," Journal of the American Chemical Society, vol. 125, no. 30, pp. 8976-8977, 2003.

[17] J.-M. Kim, Y. B. Lee, S. K. Chae, and D. J. Ahn, "Patterned color and fluorescent images with polydiacetylene supramolecules embedded in poly (vinyl alcohol) films," Advanced Functional Materials, vol. 16, no. 16, pp. 2103-2109, 2006.

[18] Y. Lu, Y. Yang, A. Sellinger et al., "Self-assembly of mesoscopically ordered chromatic polydiacetylene/silica nanocomposites," Nature, vol. 410, pp. 913-917, 2001.

[19] B. Yoon, S. Lee, and J. M. Kim, "Recent conceptual and technological advances in polydiacetylene-based supramolecular chemosensors," Chemical Society Reviews, vol. 38, pp. 19581968, 2009.

[20] R. A. Nallicheri and M. F. Rubner, "Investigations of the mechanochromic behavior of poly(urethane-diacetylene) segmented copolymers," Macromolecules, vol. 24, no. 2, pp. 517-525, 1991.

[21] R. W. Carpick, D. Y. Sasaki, and A. R. Burns, "First observation of mechanochromism at the nanometer scale," Langmuir, vol. 16, no. 3, pp. 1270-1278, 2000.

[22] M.-K. Park, K.-W. Kim, D. J. Ahn, and M.-K. Oh, "Labelfree detection of bacterial RNA using polydiacetylene-based biochip," Biosensors and Bioelectronics, vol. 35, no. 1, pp. 44-49, 2012.

[23] M. A. Reppy and B. A. Pindzola, "Biosensing with polydiacetylene materials: structures, optical properties and applications," Chemical Communications, no. 42, pp. 4317-4338, 2007.

[24] D. J. Ahn, S. Lee, and J.-M. Kim, "Rational design of conjugated polymer supramolecules with tunable colorimetric responses," Advanced Functional Materials, vol. 19, no. 10, pp. 1483-1496, 2009.

[25] Q. Huo, K. C. Russell, and R. M. Leblanc, "Chromatic studies of a polymerizable diacetylene hydrogen bonding self-assembly: a 'self-folding' process to explain the chromatic changes of polydiacetylenes," Langmuir, vol. 15, no. 11, pp. 3972-3980, 1999.

[26] H. Tanaka, M. A. Gomez, A. E. Tonelli, A. J. Lovinger, D. D. Davis, and M. Thakur, "Structural and morphological study of a melt-crystallized polydiacetylene," Macromolecules, vol. 22, no. 5, pp. 2427-2432, 1989.

[27] Y. Gu, W. Cao, L. Zhu, D. Chen, and M. Jiang, "Polymer mortar assisted self-assembly of nanocrystalline polydiacetylene bricks showing reversible thermochromism," Macromolecules, vol. 41, no. 7, pp. 2299-2303, 2008.
[28] C. Cui, G. S. Lee, and D. J. Ahn, "Composition-dependent thermochromatic reversibility of polymerized diacetylenexylenediamine complex films," Macromolecular Research, vol. 21, no. 12, pp. 1372-1374, 2013.

[29] Z.-H. Tang and B.-A. Yang, "A novel approach to polydiacetylenic nanomaterials with thermochromatic reversibility," Journal of Materials Science, vol. 42, no. 24, pp. 10133-10137, 2007.

[30] J.-M. Kim, J.-S. Lee, H. Choi, D. Sohn, and D. J. Ahn, “Rational design and in-situ FTIR analyses of colorimetrically reversibe polydiacetylene supramolecules," Macromolecules, vol. 38, no. 22, pp. 9366-9376, 2005.

[31] J. Szejtli, "Introduction and general overview of cyclodextrin chemistry," Chemical Reviews, vol. 98, no. 5, pp. 1754-1753, 1998.

[32] S. Kolusheva, T. Shahal, and R. Jelinek, "Cation-selective color sensors composed of ionophore-phospholipid-polydiacetylene mixed vesicles," Journal of the American Chemical Society, vol. 122, no. 5, pp. 776-780, 2000. 

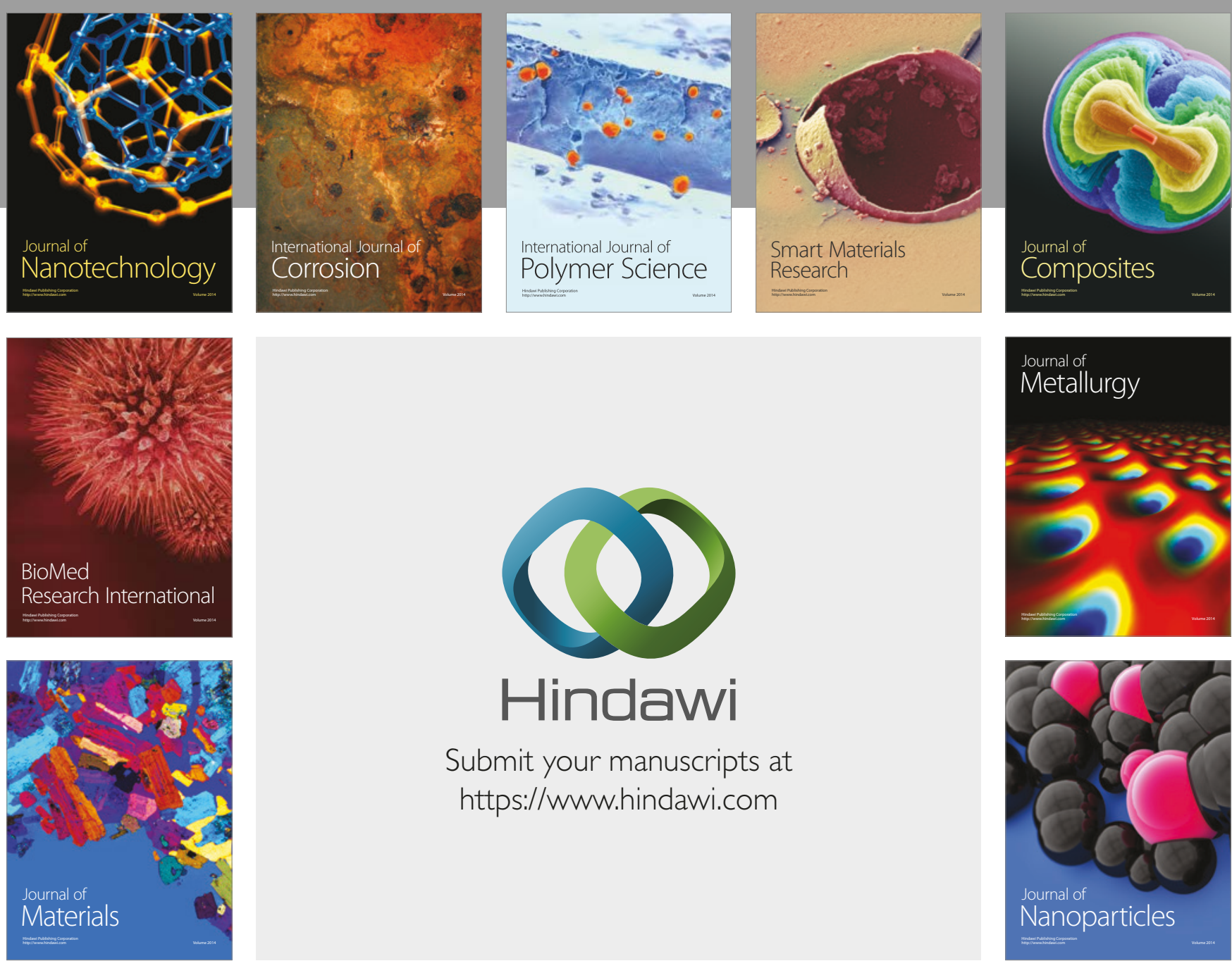

\section{Hindawi}

Submit your manuscripts at

https://www.hindawi.com
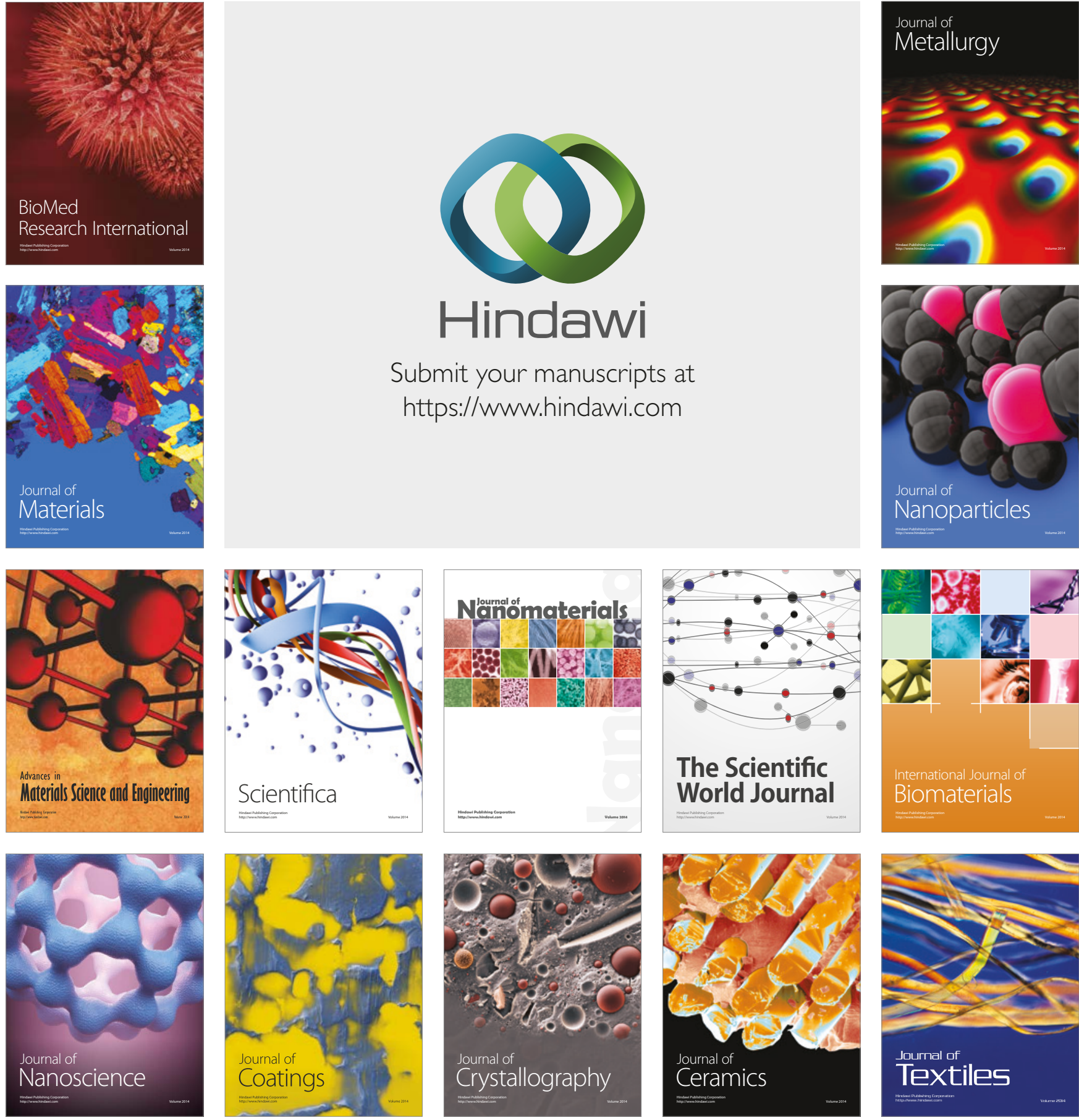

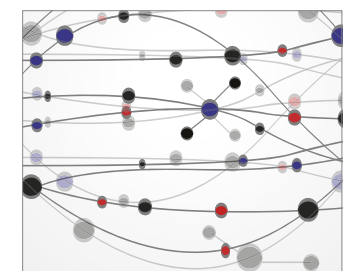

The Scientific World Journal
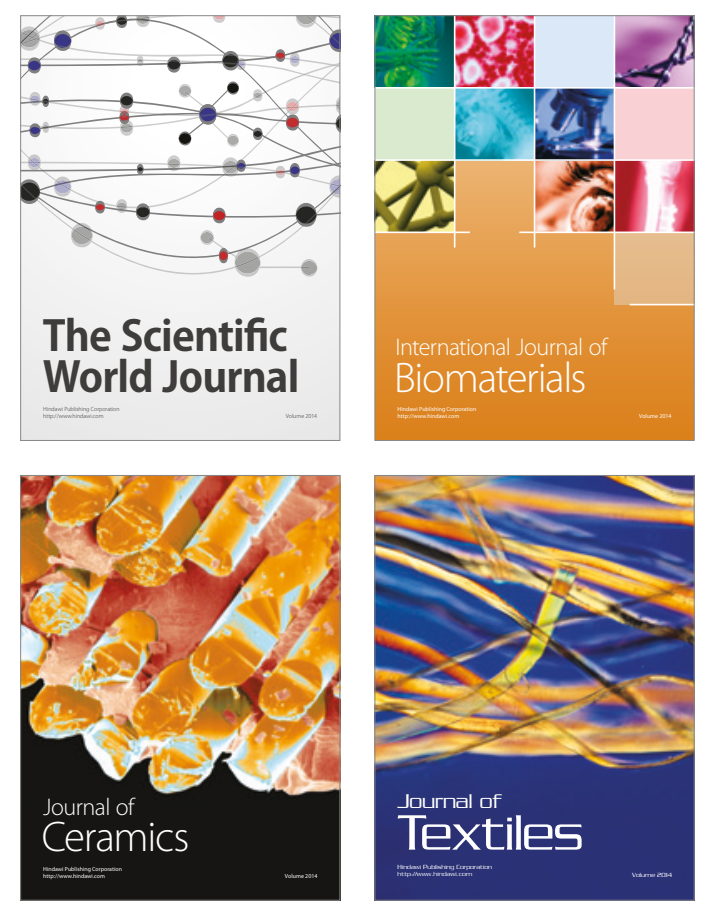